[0212-7199 (2003) 20: 4; pp 191-194] ANALES DE MEDICINA INTERNA Copyright @ 2003 ARAN EDICIONES, S.L.

AN. MED. INTERNA (Madrid) Vol. 20, N. ${ }^{\circ} 4$, pp. 191-194, 2003

\title{
Tratamiento a largo plazo de coagulación intravascular diseminada crónica con heparina de bajo peso molecular
}

\author{
J. R. GARCÍA FERNÁNDEZ, F. LÓPEZ BERENGUEL, C. AIS
}

Unidad de Hematología. Servicio Andaluz de Salud. Hospital General Básico de Baza. Granada.

\begin{abstract}
LONG-TERM TREATMENT WITH LOW MOLECULAR WEIGHT HEPARIN OF CHRONIC DISSEMINATED INTRAVASCULAR COAGULATION
\end{abstract}

\section{RESUMEN}

El termino "Coagulación intravascular diseminada" (CID) define un proceso patológico que complica la evolución clínica de diversas enfermedades, se caracteriza por la presencia de grandes cantidades de trombina y plasmina en la circulación. La lista de posibles causas de este mecanismo fisiopatológico de la enfermedad es amplia, dentro de ellas la patologia infecciosa y tumoral son las más frecuentes; entre las menos frecuentes, la existencia de un aneurisma aórtico. Describimos el caso de un paciente afecto de CID crónica con clínica hemorrágica, que en el curso del estudio etiológico se diagnosticó de un aneurisma aórtico. Se inició tratamiento con una heparina de bajo peso molecular (HBPM), pero ante la aparición de un cuadro alérgico atribuido a la misma, se modificó el tratamiento sustituyéndola por otra HBPM. Las complicaciones hemorrágicas fueron tratadas asociando fármacos antifibrinolíticos a la HBPM. El tratamiento se mantuvo durante 30 meses con una aceptable calidad de vida asociada a la aparición de efectos secundarios generalmente menores salvo el desarrollo de osteporosis clínicamente significativa.

PALABRAS CLAVE: Coagulación intravascular diseminada. Tratamiento. Heparina de bajo peso molecular. Aneurisma aórtico.

\begin{abstract}
The term "disseminated intravascular coagulation" (DIC) defined a pathologic process which complicates the clinical course of many diseases; it is characterized by huge amounts of thrombin and plasmin within the circulation. There are a lot of causes of these intermediary mechanism of disease, among these, infections and neoplasia are the most frequent. Aortic aneurysm is a vascular disease than can be complicated with DIC. We report a case of a patient affected of chronic disseminated intravascular coagulation complicated with sistemic hemorrhagic syndrome, of vascular origin (an aortic aneurysm). It was treated with a low molecular weight heparin (LMWH), but in the presence of an allergy disorder the drug was discontinued and substituted by another LMWH. The hemorraghic complications were treated with antifibrinolytics associated to the LMWH. The drug was held up 30 months with an acceptable performance status and no significant secondary effects except osteoporosis.
\end{abstract}

KEY WORDS: Disseminated intravascular coagulation. Treatment. low molecular weight heparin. Aortic aneurysm.

García Fernández JR, López Berenguel F. Tratamiento a largo plazo de coagulación intravascular diseminada crónica, con heparina de bajo peso molecular. An Med Interna (Madrid) 2003; 20: 191-194.

\section{INTRODUCCIÓN}

El término "coagulación intravascular diseminada" (CID) se refiere a un síndrome clinicopatológico en el cual se produce una activación del sistema de coagulación-fibrinolisis en todo el árbol vascular inducida por sustancias procoagulantes que son introducidas y/o producidas en la circulación sanguínea de tal forma que sobrepasan los mecanismos fisiológicos anticoagulantes (1). En el seno de este síndrome se producen grandes cantidades de trombina y plasmina intravasculares, que ocasionan isquemia tisular por microtrombos así como sangrado, debido al consumo de plaquetas y factores de la coagulación y al efecto anticoagulante de los productos de degradación del fibrinógeno-fibrina (PDF) $(2,3)$.

Existen numerosas patologías capaces de provocar un cuadro de CID, sin embargo los cuadros infecciosos y neoplasias ocasionan aproximadamente $2 / 3$ de los episodios de CID $(3,4)$.

Una de las posibles causas de CID dentro de las menos frecuentes es la existencia de un aneurisma aórtico. Aunque de rara observación, la presentación de esta asociación es bien conocida (1). Son varios los factores que predisponen a los pacientes diagnosticados de aneurisma aórtico al desarrollo de CID, fundamentalmente la existencia de una superficie vascu-

Trabajo aceptado: 27 de febrero de 2002

Correspondencia: José Ramón García Fernández. Unidad de Hematología. Hospital General Básico de Baza. Carretera de Murcia, s/n. 18800 Baza (Granada) 
lar amplia y deteriorada con placas de ateroma, y la exposición al torrente vascular de sustancias no endoteliales (5-7).

En todo paciente diagnosticado de aneurisma aórtico es importante realizar un estudio completo de coagulación incluyendo la determinación de PDF y dímeros-D, especialmente si van a ser intervenidos quirúrgicamente, ya que la existencia de una coagulopatía previa puede complicar la cirugía. Asimismo en todo paciente diagnosticado de CID sin causa evidente, debe investigarse la existencia de un aneurisma aortico (8).

Presentamos el caso de un paciente afecto de CID crónica con clínica hemorrágica, que en el curso del estudio etiológico se diagnostica de un aneurisma aórtico irresecable. Se inició tratamiento con una heparina de bajo peso molecular (HBPM) con buena evolución clínica, pero ante la aparición de un cuadro alérgico asociado a eosinofilia atribuido a la misma, se modificó el tratamiento, sustituyéndola por otra HBPM. Durante la evolución de la enfermedad presentó una CID crónica con descompensaciones periódicas, generalmente con relación a traumatismos. Estos ocasionaron complicaciones hemorrágicas que respondieron al uso de fármacos antifibrinolíticos asociados a la HBPM, salvo en una ocasión fue subsidiaria de terapia transfusional.

El interés del caso clínico se basa en la descripción de un caso de alergia a una HBPM sin reacción cruzada con otra y en el mantenimiento de un tratamiento a largo plazo con estos fármacos durante un período de 30 meses con una calidad de vida aceptable asociada a la aparición de efectos secundarios generalmente menores salvo el desarrollo de osteoporosis clínicamente significativa.

\section{CASO APORTADO}

Paciente de 75 años con antecedentes personales de artrosis e hipertensión arterial, que presenta gingivorragias, epistaxis y hematomas múltiples sin relación con traumatismos, de meses de evolución; sin historia familiar ni personal de coagulopatía.

Exploración física: constitución marfanoide, hiperextensión de articulaciones, laxitud y dedos largos, signo de Steinberg + , signo de Walter-Murdoch+, masa pulsátil en región periumbilical con pulsos periféricos conservados aunque con hipoperfusión periférica. Hematomas y petequias cutaneomucosos en diversos estadios de evolución. Resto de exploración sin hallazgos significativos.

Exploraciones complementarias:

1. Sangre periférica: hemoglobina: $11,1 \mathrm{~g} / \mathrm{dl}$. Hematocrito: 34,2\%. VCM: 96 fl. Leucocitos: 5.620/ul (fórmula leucocitaria normal). Plaquetas: 143.000/ul.

2. Estudio de coagulación: tiempo de protrombina (TP): 13,9". Actividad de protrombina: $75 \%$. Tiempo de tromboplastina parcial activada (TTPA): 41,2" (control 36"). Fibrinógeno (método de Clauss): $126 \mathrm{mg} / \mathrm{dl}$. PDF (método semicuantitativo en látex): 40-80 g/mol. Dímeros-D (técnica de enzimoinmunoanálisis ELISA): 7400 $\mathrm{ng} / \mathrm{ml}$.

3. Estudio bioquímico: urea $60 \mathrm{mg} / \mathrm{dl}$. Creatinina: $1,4 \mathrm{mg} / \mathrm{dl}$. Perfil hepático normal. Metabolismo ferrocinético normal.

4. Serologías virales: negativas.

5. TAC abdominal: aneurisma de aorta abdominal de $13 \mathrm{~cm}$ de longitud y diámetro máximo de $6,4 \mathrm{~cm}$, que engloba arterias renales, con afectación funcional del riñón izquierdo.

Tras el diagnóstico de síndrome de Marfan, aneurisma de aorta abdominal irresecable y CID crónica, se inició tratamiento con HBPM: nadroparina $0,4 \mathrm{ml} / 12$ horas (equivalente a $3.800 \mathrm{UI}$ antiXa) con buena respuesta clínica medida en términos de desaparición de los hematomas cutáneo-mucosos, así como aceptable respuesta biológica: aumento de la cifra de fibrinógeno sérico y plaquetas, y disminución del nivel de PDF y Dímeros-D.

Dos meses después presentó una clínica de prurito generalizado inicialmente, que se complicó con la aparición de máculas eritematoso-pruriginosas en glúteos y región inguinal de evolución autolimitada, asociado a eosinofilia tanto relativa (25\%) como absoluta $(1.450 / \mu 1)$. Se realizó estudio alérgico en el que se practicaron pruebas epicutáneas en parche según una batería estándar que resultaron negativas; Así como un estudio del fármaco más probablemente relacionado con la clínica (nadroparina); las pruebas cutáneas (Prick-test e intradermoreacción) en lectura inmediata y tardía resultaron negativas, así como las pruebas epicutáneas. Aún así, sobre la base del comportamiento clínico se atribuyó al tratamiento con HBPM, y se sustituyó por otra: enoxaparina, con lo cual desapareció el cuadro alérgico y se mantuvo el control del cuadro de CID.

Inicialmente se controló de forma aceptable con una dosis de enoxaparina de $40 \mathrm{mg} / \mathrm{día}$ (4.000 UI anti-Xa) pero ante el ascenso de las cifras de dímeros-D con reaparición de la clínica hemorrágica, se aumentó la dosis a $60 \mathrm{mg} /$ día (6.000 UI anti-Xa) con lo que se normalizaron los índices de fibrinolisis. El paciente mantuvo de forma crónica y con variaciones fisiopatológicas el siguiente estudio de sangre periférica: hemoglobina: 12,4 g/dl, Hematocrito: 39,9\%, VCM: 92,7 fl, Leucocitos: 5840/ul (fórmula leucocitaria normal), plaquetas: 147.000/ul; y estudio de coagulación: tiempo de protrombina: 12 ", actividad de protrombina: $+100 \%$, tiempo de tromboplastina parcial activada: 35,7" (control: 37,6"), fibrinógeno: $360 \mathrm{mg} / \mathrm{dl}$ y Dímeros-D (técnica de ELISA) oscilando entre 850 y $4600 \mathrm{ng} / \mathrm{ml}$ (Tabla I). La situación se mantuvo como una CID crónica controlada, aunque ante diversas incidencias: infecciones dentarias, traumatismos mínimos, cuadros febriles,... se produjo clínica hemorrágica de entidad variable. En el control de estos episodios se demostró eficaz el tratamiento con agentes antifibrinolíticos (ácido épsilon amino caproico a dosis de 4 gramos cada 8 horas por vía oral) asociados al tratamiento con enoxaparina mientras persistía el problema hemorrágico. Aunque precisó transfusión de hemoderivados (concentrados de hematíes) en una ocasión debido a un hematoma severo en miembro inferior derecho secundario a traumatismo.

\section{TABLA I}

ESTUDIO BÁSICO DE COAGULACIÓN AL DIAGNÓSTICO Y DURANTE EL TRATAM IENTO EN FASE ESTABLE

\begin{tabular}{lll}
\hline & Pre-tratamiento & Pos-tratamiento \\
\hline TTPA & $41,2^{\prime \prime}$ (control: 36") & $35,7^{\prime \prime}$ (control: 37,6" ) \\
TP & $13,9^{\prime \prime}$ & $12^{\prime \prime}$ \\
Act. de protrombina & $75 \%$ & $+100 \%$ \\
Fibrinógeno & $126 \mathrm{mg} / \mathrm{dl}$ & $360 \mathrm{mg} / \mathrm{dl}$ \\
PDF & $40-80 \mu \mathrm{g} / \mathrm{ml}$ & $<10 \mu \mathrm{gl} / \mathrm{ml}$ \\
Dímero-D & $7.400 \mathrm{ng} / \mathrm{ml}$ & $850-4.600 \mathrm{ng} / \mathrm{ml}$ \\
Plaquetas & $143.000 / \mu \mathrm{l}$ & $147.000 \mu \mathrm{l}$ \\
\hline
\end{tabular}

El tratamiento a largo plazo con HBPM en este caso ha presentado efectos secundarios menores derivados de su administración parenteral crónica como son: hematomas en el lugar de inyección, nódulos subcutáneos, dolor cutáneo leve-moderado y atrofia cutánea. Como efecto secundario "mayor" desarrolló osteoporosis clínicamente significativa, con aplastamiento traumatico-osteoporótico a nivel de las vértebras L3, L1 y D12, con dificultad para la deambulación y movilidad del paciente aunque sin presentar afectación neurológica. Asimismo presentó clínica hemorrágica, que en cualquier caso no se puede atribuir al tratamiento con HBPM sino a la coagulopatía de base. El paciente falleció por un infarto agudo de miocardio a los 30 meses de iniciado el proceso. 


\section{DISCUSIÓN}

El abordaje tanto diagnóstico como terapéutico de la coagulación intravascular diseminada nos introduce en un campo confuso $(9,10)$, ya que sabemos que la presencia de CID es una manifestación de la enfermedad como lo son la fiebre, la hiponatremia o el dolor abdominal, más que una enfermedad concreta (8). Existen numerosas patologías capaces de provocar un cuadro de CID $(1,3,8,9,11)$ y por otra parte las posibilidades de nuestro arsenal terapéutico en este caso son cuando menos limitadas (12-16); esta limitación se hace aún más patente si el proceso de base que provoca el cuadro clínico no tiene tratamiento específico, como en el caso que nos ocupa.

Una de las posibles causas de CID es la existencia de un aneurisma aórtico, la existencia de esta asociación está bien documentada. En una serie de pacientes con aneurisma aórtico, aunque el $40 \%$ de los mismos presentaba productos de degradación del fibrinógeno-fibrina (PDF) elevados, solo un 4\% de los mismos presentaba un cuadro clínico característico (1). Son varios los factores que predisponen a los pacientes diagnosticados de aneurisma aórtico al desarrollo de CID, fundamentalmente la existencia de una superficie vascular amplia y deteriorada con placas de ateroma y exposición al torrente vascular de sustancias no endoteliales (1).

En todo paciente diagnosticado de aneurisma aórtico es importante realizar un estudio completo de coagulación incluyendo la determinación de PDF y dímeros-D, especialmente si van a ser intervenidos quirúrgicamente, ya que la existencia de una coagulopatía previa puede complicar la cirugía. Asimismo en todo paciente diagnosticado de CID sin causa aparente, debe investigarse la existencia de un aneurisma aórtico.

La iniciación de la activación del sistema de la coagulación-fibrinolisis se atribuye en estos casos a una activación de la vía intrínseca de la coagulación debido a la exposición de endotelio dañado a la circulación sanguínea (17), así como a una activación del sistema extrínseco por la existencia de grandes cantidades de factor tisular existentes en las placas de ateroma, que suelen ser el factor predisponente más importante en la génesis de los aneurismas aórticos (18). Se han descrito casos de corrección espontánea de dicha coagulopatía atribuida a la formación de un coágulo intraaneurismático que evita el acceso de la sangre a su interior y por tanto la activa- ción subsiguiente del sistema de la coagulación-fibrinolisis (1).

El único tratamiento de base capaz de curar este proceso es la resección quirúrgica del aneurisma. Para realizar esta es necesario, aparte de las medidas de soporte vital básico necesarias, tratar previamente la coagulopatía. Esta puede ser corregida con transfusiones de hemoderivados (fundamentalmente plasma fresco congelado y/o concentrados de plaquetas) e infusión contínua de heparina IV (1), este tratamiento es necesario en pacientes con clínica hemorrágica, aunque por razones obvias no es operativo a largo plazo.

Las heparinas de bajo peso molecular son fracciones de heparina con peso molecular comprendido entre 4.000 y 8.000 dalton. Se diferencian de la heparina no fraccionada en la ratio: actividad anti-Xa/ actividad anti-IIa, que es siempre superior a 1:1, por lo que se postula que presentan un menor riesgo hemorrágico con el mismo potencial antitrombótico. Se han descrito como tratamiento efectivo en diversos estudios no controlados $(19,20)$ y en otro multicéntrico randomizado (21), en este último únicamente se pudo demostrar una menor incidencia de complicaciones hemorrágicas en comparación con el uso de heparina no fraccionada, no así una mayor supervivencia (4). En base a estos resultados las heparinas de bajo peso molecular son una buena opción terapéutica en tratamientos a largo plazo por diversas razones: su comodidad de uso (una vez al día y vía subcutánea), la posibilidad de autoadministración, no existir necesidad de controles frecuentes y la baja incidencia de complicaciones hemorrágicas y/o trombopenia inducida por heparina.

El tratamiento inicial con nadroparina fue efectivo en términos de control de la CID, aunque se produjo una reacción alérgica que imposibilitó el mantenimiento del mismo. La continuación del mismo con otra HBPM (enoxaparina) controló el cuadro de CID y fue efectivo con modificaciones puntuales de dosis en función de la evolución clínico-biologica; aumentando la dosis en presencia de clínica hemorrágica y/o aumento de la cifra de dímeros-D, a la vez que se asociaban antifibrinolíticos en presencia de clínica hemorrágica severa, únicamente durante el episodio agudo. La utilización de estos últimos fármacos no se asoció a clínica trombótica en ningún caso, posiblemente porque se utilizaron de forma puntual y siempre asociados a HBPM.

\section{Bibliografía}

1. Seligsohn U. Disseminated intravascular coagulation. In; Bbeutler E, Lichtman MA, Coller BS, Kipps TJ (editors). Hematology. New York: McGraw-Hill Inc; 1995. p.1497-516.

2. Staudinger T, Locker GJ, Frass M. Management of acquired coagulation disorders in emergency and intensive-care medicine. Semin Thromb Hemost 1996; 22: 93-104.

3. Rocha E, Paramo JA, Montes R, Panizo C. Acute generalized, widespread bleeding. Diagnosis and management. Haematologica 1998; 83: 1024-37.

4. de Jonge E, Levi M, Stoutenbeek CP, van Deventer SJ. Current drug treatment strategies for disseminated intravascular coagulation. Drugs 1998; 55: 767-77.

5. Riewald M, Riess $H$. Treatment options for clinically recognized disseminated intravascular coagulation. Semin Thromb Hemost 1198; 24: 53-9.

6. Amstutz P, Moyo JS. Disseminated intravascular coagulations. Cah

Anesthesiol 1996; 44: 219-28.

7. Carey MJ, Rodgers GM. Disseminated intravascular coagulation: clinical and laboratory aspects. Am J Hematol 1998; 59: 65-73.

8. Kitchens CS. Disseminated intravascular coagulation. Curr Op Hematol 1995; 2 : 402-6.

9. Colman RW, Robboy SJ, Minna JD.. Disseminated intravvascular coagulation: a reappraisal. Annu Rev Med 1979; 30: 359-74.

10. Spero JA, Lewis JH, Hsiba V. Disseminated intravascular coagulation Findings in 346 patients. Thromb Haemostas 1980; 43: 28-33.

11. Bauer K, ten Cate H, Barzegar S, Spriggs DR, Sherman ML, Rosenberg $\mathrm{RD}$. Tumor necrosis factor infusions have a procoagulant effect on the hemostatic mechanism of humans. Blood 1989; 74: 165-72.

12. Feinstein D. Diagnosis and management of disseminated intravascular coagulation: the role of heparin therapy. Blood 1982; 86: 284-7.

13. Vinazzer HA. Antithrombin III in shock and disseminated intravascular coagulation. Clin Appl Thromb 1995; 12: 62-5. 
14. Ziegler EJ, McCutchan JA, Fierer J. Treatment of gram-negative bacteremia and shock with human antiserum to a mutant Esherichia coli. N Engl J Med 1982; 307: 1225-30.

15. Fisher CJ, Agosti JM, Opal SM. Treatment of septic shock with the tumor necrosis factor receptor:Fc fusion protein. N Engl J Med 1996; 334: $1697-702$.

16. Warrel R, Kempin S. Treatment of severe coagulopathy in the Kasabach-Merrit syndrome with aminocaproic acid and cryoprecipitate. N Engl J Med 1985; 313: 309-12.

17. Fine NL, Applebaum J, Elguezabal A, Castleman L. Multiple coagulation defects in association with dissecting aneurysm. Arch Intern Med 1967; 119: 522-6.

18. Wilcox JN, Smith KM, Schwartz SM, Gordon D. Localization of tissue factor in the normal vessel and in the atherosclerotic plaque . Proc Natl Acad Sci USA 1989; 86: 2839-42.

19. Audibert G, Lambert H, Toulemonde F. Utilisation d'une héparine de bas poids moléculaire, la CY 222, dans le traitment des coagulopathies de consommation. J Mal Vasc 1987; 12 Supl. B: 147-51.

20. Gillis S, Dann EJ, Eldor A. Low molecular weight heparin in the prophylaxis and treatment of disseminated intravascular coagulation in acute promyelocytic leukemia . Eur J Haematol 1995; 54: 59-60.

21. Sakuragawa N, Hasegawa H, Maki M, Oguma Y, Nakagawa M. Clinical evaluation of low-molecular-weight-heparin (FR-860) on disseminated intravascular coagulation (DIC)- a multicenter co-operative double-blind trial in comparison with heparin. Thromb Res 1993; 72: 475-500. 